\title{
Abordagem cirúrgica de lesão granulomatosa criptocócica cerebral (criptococoma) em paciente imunocompetente
}

\section{Surgical Approach to an Immunocompetent Patient's Cerebral Granulomatosis (cryptococcoma)}

\author{
Marco Aurélio Moscatelli Alvarenga ${ }^{1}$ Antônio Augusto Roth Vargas ${ }^{2}$ Marcelo Senna Xavier de Lima ${ }^{3}$ \\ Paulo Roland Kaleff ${ }^{3}$ Luciano de Souza Queiroz ${ }^{4}$ Cícero Ronaldo Ferreira da Silva ${ }^{1}$ \\ Sinval Malheiros Pinto Neto ${ }^{5}$ José Alberto Pereira Pires ${ }^{1}$
}

\footnotetext{
${ }^{1}$ Neurocirurgião da Fundação Centro Médico Campinas, Campinas, SP, Brasil

2 Professor Chefe do Departamento de Neurocirurgia da Fundação Centro Médico Campinas e da Santa Casa de Misericórdia de Limeira, Limeira, SP, Brasil

${ }^{3}$ Professor Adjunto do Departamento de Neurocirurgia da Fundação Centro Médico Campinas e da Santa Casa de Misericórdia de Limeira, Limeira, SP, Brasil

${ }^{4}$ Professor Doutor do Departamento de Anatomia Patológica da Faculdade de Ciências Médicas da Universidade de Campinas (Unicamp), Campinas, SP, Brasil

${ }^{5}$ Residente de Neurocirurgia da Santa Casa de Misericórdia de Limeira, Limeira, SP, Brasil
}

Arq Bras Neurocir 2015;34:73-78.

Address for correspondence Marco Aurélio Moscatelli Alvarenga, MD, Av. Engenheiro Roberto Freire, 9.036/1.003, Ponta Negra, Natal, RN, Brasil, CEP 59090-000 (e-mail: marcomosca13@hotmail.com).

Fundação Centro Médico Campinas, Campinas, SP, Brasil e Santa Casa de Misericórdia de Limeira, Limeira, SP, Brasil

\section{Resumo \\ Palavras-chave \\ - criptococose \\ - meningite criptocócica \\ - cryptococcus neoformans}

A criptococose é a principal micose que atinge o sistema nervoso central, sendo de distribuição mundial, mais prevalente em países em desenvolvimento. Atualmente, são descritas duas subespécies: Cryptococcus neoformans neoformans, associado à imunossupressão, e Cryptococcus neoformans gattii, em pacientes imunocompetentes. A criptococose do sistema nervoso central parenquimatosa em sua forma granulomatosa, ou criptococoma, é extremamente rara, sendo mais frequente a forma meningítica ou meningoencefalítica.

Relatamos o caso de um paciente de 35 anos, não portador da síndrome de imunodeficiência adquirida e imunocompetente, com manifestações clínicas iniciais de cefaleia crônica, hemiparesia progressiva, confusão mental aguda e primeiro episódio de crise convulsiva tônico-clônica generalizada. Hipóteses diagnósticas préoperatórias de neoplasia maligna ou abscesso cerebral. Realizado procedimento cirúrgico, remoção completa da lesão maior, diminuindo o efeito de massa. A lesão foi diagnosticada, através de exame anatomopatológico, como criptococoma, o que torna o caso extremamente raro pelo tamanho da lesão e por ser o paciente imunocompetente. Houve melhora completa do déficit neurológico. O paciente encontra-se em seguimento clínico e tratamento medicamentoso com anfotericina-B. received

September 9, 2013 accepted

November 1, 2014
DOI http://dx.doi.org/ 10.1055/s-0035-1552899. ISSN 0103-5355.
Copyright $@ 2015$ by Thieme Publicações License terms Ltda, Rio de Janeiro, Brazil 


\begin{abstract}
Keywords

- cryptococcosis

- meningitis cryptococcal

- cryptococcus neoformans

Cryptococcosis is the main mycosis affecting the central nervous system, with worldwide distribution and higher prevalence in developing countries. Currently, two subspecies are described: Cryptococcus neoformans neoformans (associated with immunosuppression) and Cryptococcus neoformans gattii (in immunocompetent patients). Cryptococcosis of the central nervous system as a parenchymal granuloma or cryptococcoma is extremely rare, contrasting to the much more frequent meningitic form. We report a 35 year-old immunocompetent patient with chronic headache, progressive hemiparesis, acute confusion and first episode of tonic-clonic seizures. Preoperative diagnostic hypotheses were malignancy or abscess. A large mass $(5.5 \mathrm{~cm})$ was surgically removed from the right parietal lobe relieving the mass effect. This was later diagnosed by pathological examination as cryptococcoma, an extremely rare case in view of lesion size and its occurrence in an immunocompetent subject. The patient evolved with complete resolution of the neurological deficit and is on amphotericin-B treatment.
\end{abstract}

\section{Introdução}

Criptococose (torulose, blastomicose europeia, doença de Busse-Buschke) é uma micose de origem sistêmica, com porta de entrada inalatória, causada por fungos do complexo Cryptococcus neoformans, atualmente com duas subespécies: Cryptococcus $n$. neoformans e Cryptococcus $n$. gattii. ${ }^{1,2}$

Esta micose abrange dois tipos distintos do ponto de vista clínico e epidemiológico: (1) criptococose oportunista, urbana, associada a condições de imunodepressão celular causada predominantemente por Cryptococcus neoformans, frequentemente relaciona-se a habitat de aves, excretas secas, ricas em fontes de nitrogênio, como ureia e creatinina; condições favoráveis ao crescimento abundante desta levedura formam microfocos, notadamente em centros urbanos e relacionados a pombos; ${ }^{3}$ (2) criptococose primária, de hospedeiro aparentemente imunocompetente, endêmica em áreas tropicais e subtropicais, causada predominantemente por Cryptococcus gattii. Ambas causam meningoencefalite, de evolução grave e fatal, acompanhada ou não de lesão pulmonar evidente, fungemia e focos secundários na pele, ossos, rins, suprarrenal, cerebral entre outros. No momento do diagnóstico encefálico, a lesão pulmonar já não é encontrada. A possível propensão desses organismos para infectarem o sistema nervoso central talvez seja pelos ricos substratos para a fenol oxidase, como a dopamina cerebral. ${ }^{4}$

A mortalidade por criptococose é estimada em $10 \%$ nos países desenvolvidos, chegando a $43 \%$ nos países em desenvolvimento em um tempo médio de sobrevida de 14 dias. $^{5}$

\section{Relato do Caso}

Paciente masculino de 35 anos, usuário de drogas, com história de cefaleia holocraniana em evolução há 30 dias sem investigações prévias. Após 15 dias do início do quadro, evolui com hemiparesia proporcionada incompleta à esquerda progressiva associada à desorientação e confusão mental. Apresentou primeiro episódio de crise convulsiva tônico-clônica generalizada. Após estabilização clinica, foi realizada investigação radiológica com tomografia computadorizada (TC) de crânio com contraste (-Fig. 1), notando-se duas lesões expansivas cerebrais em região parietotemporal direita com captação perilesional de contraste endovenoso e sinais radiológicos de hipertensão intracraniana. 0 paciente manteve confusão mental com quadro clinico estável após o uso de corticosteroides. Foi realizada ressonância magnética (RM) de encéfalo ( - Fig. 2) para programação de abordagem cirúrgica eletiva com hipóteses diagnósticas de abscesso ou tumor.

Foi realizado procedimento cirúrgico sem intercorrências com ressecção da lesão maior parietal em sua totalidade. O paciente no pós-operatório apresentou melhora completa do déficit neurológico e confusão mental (-Fig. 3). Após o diagnóstico anatomopatológico, de lesão criptocócica, iniciou-se terapia antifúngica com anfotericina B. Foi realizada TC de crânio após 30 dias, verificou-se melhora do edema perilesional e ausência de lesão (-Fig. 4).

\section{Aspectos Radiológicos da Criptococose Cerebral}

Os seguintes aspectos podem ser observados em TC de crânio ou RM de encéfalo nos pacientes com criptococose do sistema nervoso central: espaços de Virchow-Robin (VR) dilatados, pseudocistos (também chamados de "pseudocistos gelatinosos" e "bolhas de sabão", correspondem à proliferação de fungos em espaços de VR invadindo o parênquima circundante ou em espaços de VR confluentes), nódulos intracerebrais ou massas (também chamados de lesões nodulares, granulomas, criptococoma ou 


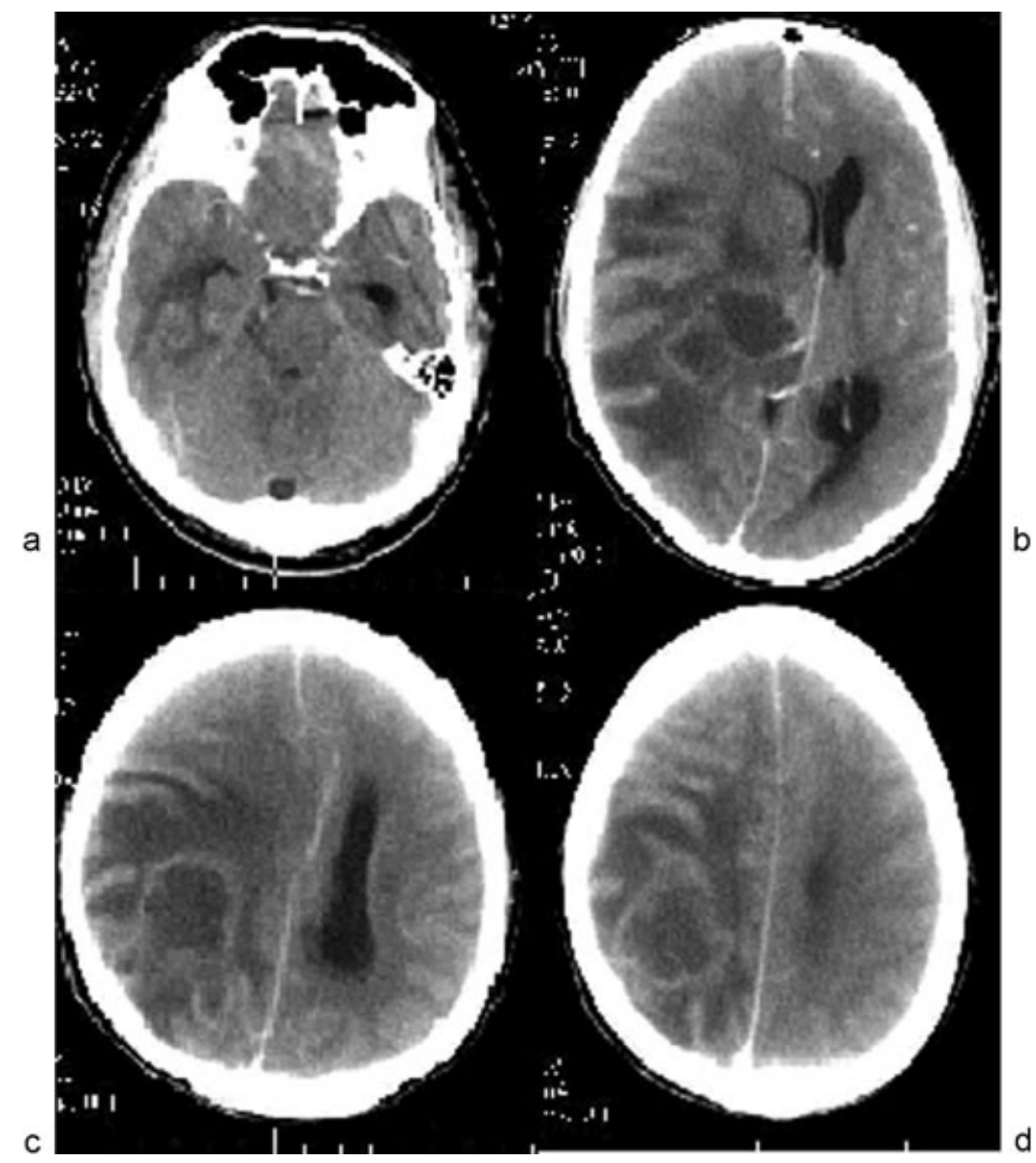

Fig. 1 Tomografia computadorizada de crânio pré-operatória com contraste. Lesão na substância branca profunda nos lobos parietal e temporal direitos, hipodensa, com impregnação periférica pelo contraste iodado, e intenso edema perilesional.

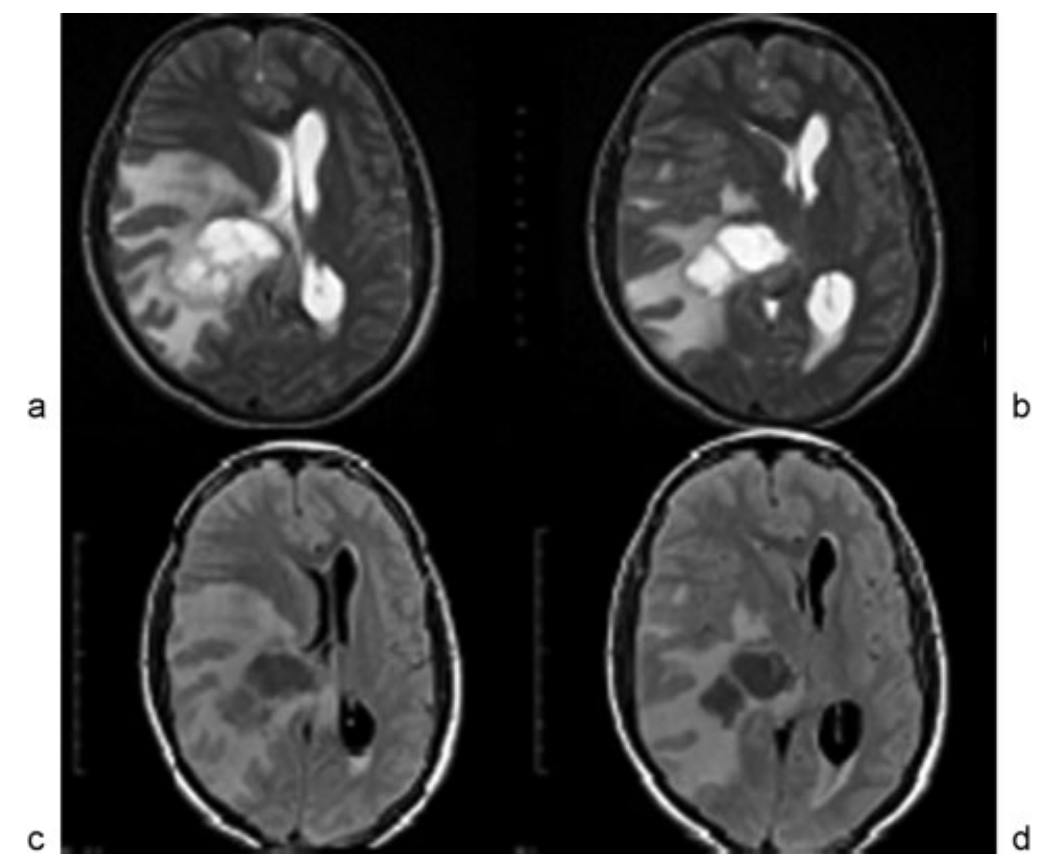

Fig. 2 Ressonância magnética (RM) de encéfalo em ponderação T2 - (a) e (b). RM de encéfalo em ponderação T2 FLAIR - (c) e (d). Os exames mostram lesão bem delimitada, altamente hidratada (hipersinal em T2 e supressão parcial do sinal em FLAIR), com edema da substância branca limítrofe, forte efeito de massa, desvio da linha média e hidrocefalia contralateral por compressão do terceiro ventrículo. 


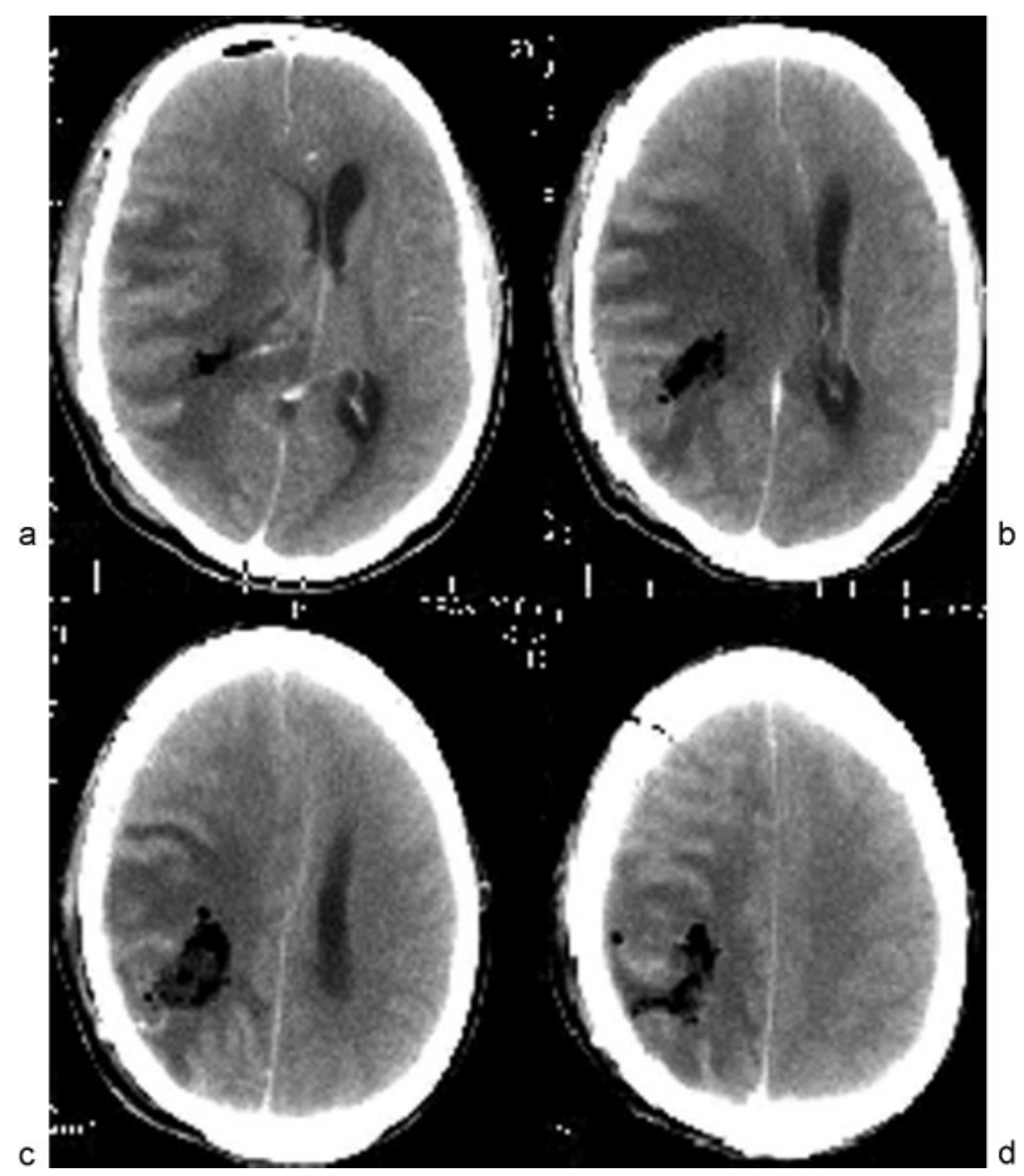

Fig. 3 Tomografia computadorizada (TC) de crânio pós-operatória imediata sem contraste - (a) e (b). TC de crânio pós-operatória imediata com contraste - (c) e (d). Os exames mostram remoção total da lesão e pneumoencéfalo residual no leito cirúrgico.

abscessos cerebrais fúngicos) e hidrocefalia. As seguintes lesões podem ser consideradas como sequelas neurológicas: infartos isquêmicos, calcificação sem edema periférico ou efeito de massa com ausência de realce com contraste intravenoso ${ }^{6}$ (-Fig. 5).

\section{Discussão}

A criptococose é a principal micose que atinge o sistema nervoso central, sendo de distribuição mundial, mais prevalente em países em desenvolvimento e principalmente em pacientes imunossuprimidos. Porém, no caso descrito acima, encontramos uma apresentação rara, acometendo paciente imunocompetente, em sua forma granulomatosa. Cefaleia, confusão mental e crise convulsiva são os principais sintomas encontrados em pacientes com criptococoma cerebral, também vistos em diversas lesões cerebrais como meningites, hemorragias, traumas cranioencefálicos, entre outras.

Após a realização de tomografia computadorizada com contraste, foi notado grande processo expansivo intraparenquimatoso com efeito de massa, grande área de edema vasogênico, desvio de linha média e captação de contraste. Foram aventadas as hipóteses diagnósticas de abscesso cerebral e glioblastoma multiforme. Após ressonância magnética encefálica, realizou-se exérese da lesão expansiva por acesso parietal posterior após confirmação com neuronavegador, o que tornou ainda mais precisa a via de acesso, pois a lesão possuía íntimas relações com a área cortical motora direita. Após a corticectomia, notamos uma massa endurecida de limites precisos, sem sangramento lesional e sem sinais de infiltrações, colocando em dúvida o diagnóstico de glioblastoma multiforme, porém prosseguimos com a resseç̧ão completa da lesão em bloco. 


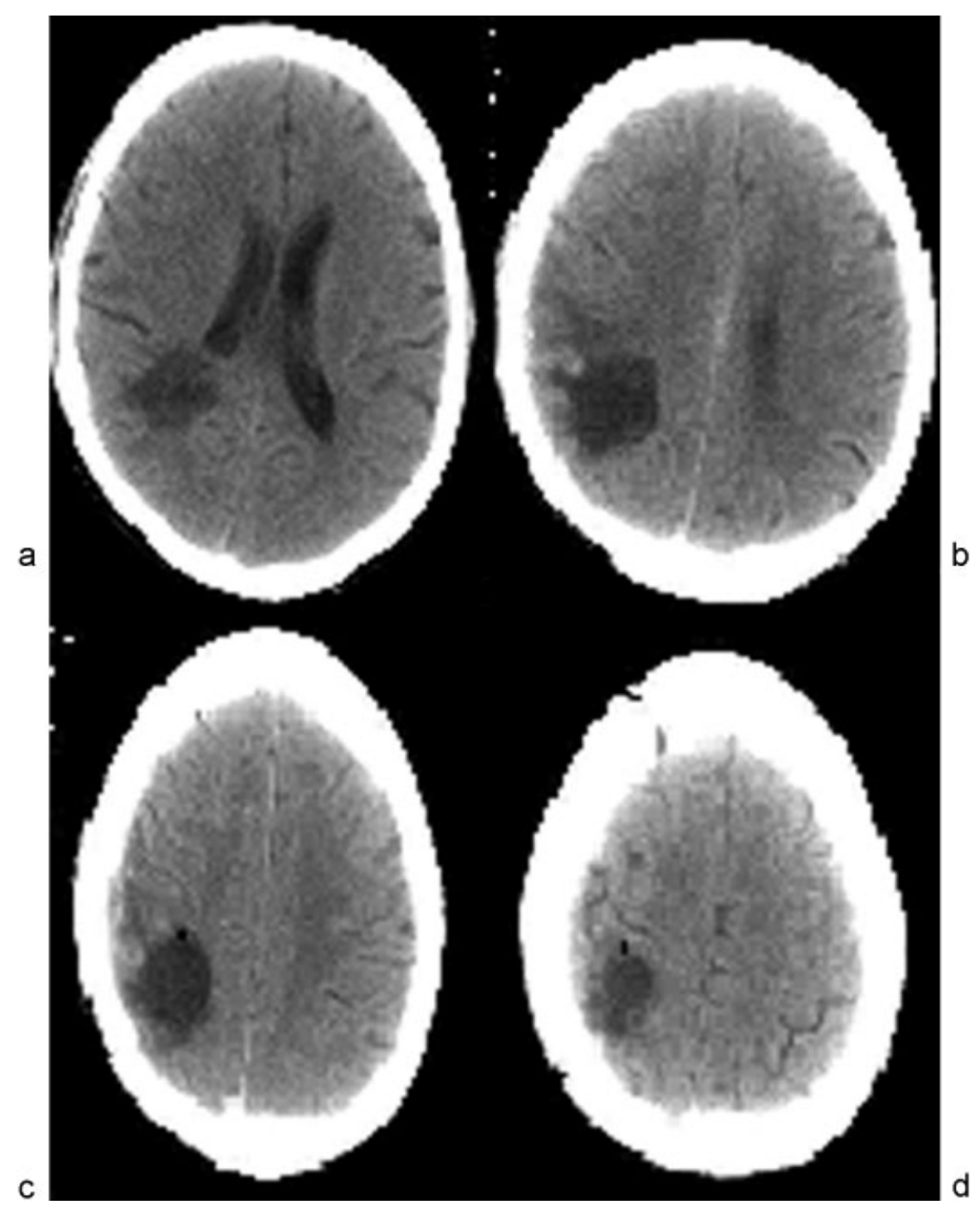

Fig. 4 Tomografia computadorizada (TC) de crânio pós-operatória tardia (30 dias) sem contraste - (a) e (b). TC de crânio pós-operatória tardia (30 dias) com contraste - (c) e (d).

Paciente foi extubado em sala cirúrgica, com melhora completa do quadro de hemiparesia após 24 horas, consciente e orientado em tempo e espaço, com discreta cefaleia pós-operatória.

O diagnóstico anatomopatológico foi criptococose cerebral em sua forma granulomatosa (criptococoma). Imediatamente, iniciamos tratamento com anfotericina $B$ e mantivemos 0 paciente em internação hospitalar. A tomografia computadorizada após 30 dias mostrou ressecção completa da lesão, sem recidivas, e diminuição da lesão temporal remanescente.

Este relato de caso mostra que, principalmente em países em desenvolvimento e apesar de extremamente rara, temos que levar em consideração a criptococose como diagnóstico diferencial de lesões expansivas cerebrais em pacientes imunocompetentes. Neste caso, a indicação cirúrgica inicial deveu-se ao grande efeito de massa da lesão e à necessidade de diagnóstico etiológico. Seguiuse terapia adjuvante por anfotericina B com melhora completa do déficit neurológico já no pós-operatório imediato.

Conflitos de Interesse

Os autores declaram não haver conflitos de interesse. 


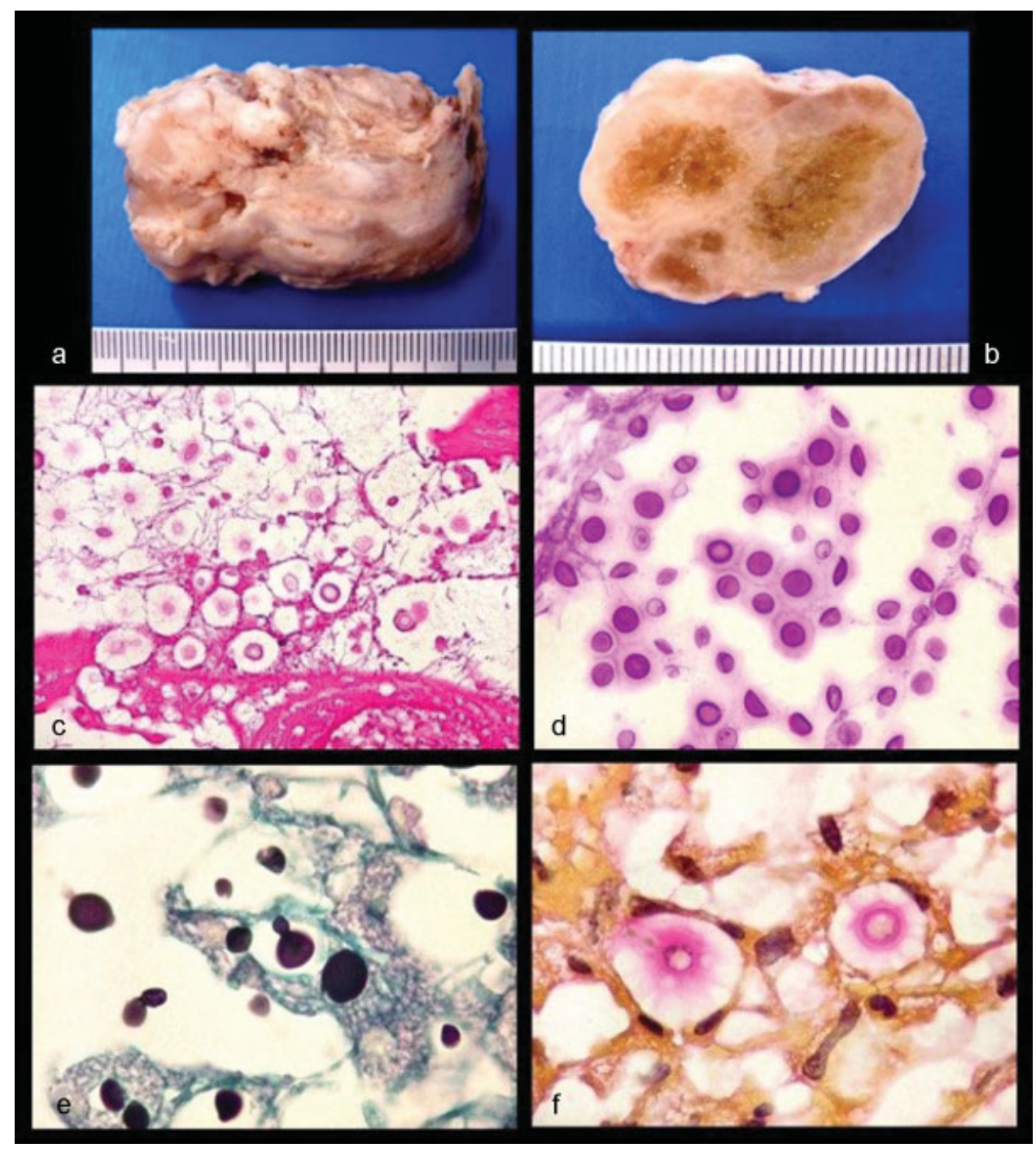

Fig. 5 Anatomia patológica. Espécime cirúrgico - (a) e (b): mediu 5,5 cm no maior diâmetro, apresentava boa delimitação, superfície externa levemente irregular e consistência firme; ao corte, havia uma cápsula fibrosa em toda a volta e conteúdo de consistência gelatinosa. HE - (c): abundantes fungos compatíveis com Cryptococcus neoformans, corpo celular redondo e cápsula espessa incolor, em meio a material filamentoso ou grumoso interpretado como fibrina (200x). PAS - (d): fungos em forma de leveduras, justapostos, com parede celular fortemente PAS positiva, cápsula mucopolissacarídica mais fracamente reagente; ausência de reação inflamatória (200x). Grocott - (e): parede celular dos fungos impregna-se em negro pela prata; cápsula mucopolissacarídica não se marca; ao centro, levedura em brotamento simples (400x). Mucicarmim (f): fungos em meio a macrófagos, alguns xantomatosos; ausência de reação granulomatosa (400x).

\section{Referências}

1 Charlier C, Dromer F, Lévêque C, et al. Cryptococcal neuroradiological lesions correlate with severity during cryptococcal meningoencephalitis in HIV-positive patients in the HAART era. PLoS One 2008;3(4):e1950

2 Rodrigues ML, Alviano CS, Travassos LR. Pathogenicity of Cryptococcus neoformans: virulence factors and immunological mechanisms. Microbes Infect 1999;1(4):293-301

3 Perfect JR, Casadevall A. Cryptococcosis. Infect Dis Clin North Am 2002;16(4):837-874
4 Moretti ML, Resende MR, Lazéra MS, Colombo AL, ShikanaiYasuda MA. Guidelines in cryptococcosis-2008. Rev Soc Bras Med Trop 2008;41(5):524-544

5 Casadevall A, Perfect JR. Cryptococcus neoformans. Washington: ASM Press; 1998

6 Kwon-Chung KJ, Bennett JE. Epidemiologic differences between the two varieties of Cryptococcus neoformans. Am J Epidemiol $1984 ; 120(1): 123-130$ 